\title{
Adolescent Behavior: Multiple Levels of Social Influence
}

\author{
J. R. Temple
}

Published online: 17 August 2012

(C) Springer Science+Business Media, LLC 2012

Adolescence is a critically important time period characterized by identity development and formation, increased autonomy and peer influence, biological changes, and exposure to new environments and behavior. Our knowledge of this key developmental timeframe has blossomed over the previous three decades with the help of well-designed longitudinal studies and an increased understanding of factors relevant to positive and negative development. Studies have revealed that exposure to a range of risk factors is strongly predictive of current and later psychosocial problems. Although not as well understood, we also know that protective factors can buffer or moderate the effect of these risk factors. Indeed, one variable that has consistently been associated with positive development is an attachment to prosocial role models (Yancey, Siegel, \& McDaniel, 2002). To that end, the primary focus of this double issue is on the importance and influence of parents and other adults in reducing adolescent risk behavior and promoting positive youth development.

Two studies in this issue focus on adolescent substance use, specifically on the role of parents in preventing or contributing to this behavior. In a sample of at-risk adolescents attending alternative high school, Heddy Clark and colleagues sought to determine

J. R. Temple ( $\square)$

Department of Obstetrics and Gynecology, University of Texas Medical Branch, Galveston, TX, USA

e-mail: jetemple@utmb.edu whether parental monitoring protected against the use of a range of substances over time. Notably, they found that even among this high-risk sample, adolescents with higher parental monitoring at baseline evidenced lower use of alcohol, marijuana, prescription drugs, and other hard drugs relative to their adolescent counterparts with less parental monitoring. The authors also observed reduced substance use 2 years after baseline in those with higher parental monitoring. This study illustrates the critical importance of including parental monitoring as a component in drug prevention programs. The findings are consistent with a growing body of research (Lac \& Crano, 2009) and lend credibility to the Parents: The Anti-Drug campaign currently being implemented by the Office of National Drug Control Policy.

The study by Bettina Friese and colleagues considers a specific role parents have in moderating adolescent substance use. Supported by the knowledge that access to and availability of substances is a strong predictor of adolescent substance use (Dent, Grube, \& Biglan, 2005), the authors conducted in-depth qualitative interviews of parents of adolescents to determine how parents store and monitor alcohol in their home. This study reveals the overall difficulty parents face in limiting access to alcohol and indicates that parents' trust of their adolescent is the primary safeguard against such access. Thus, the authors rightly state that intervention programs aimed at parents should frame the need to secure alcohol at home as a matter of safety as opposed to a matter of trust between parents and teens. 
Notably, the method of communicating parenting strategies to increase parental knowledge is consistent with another study published in this issue (Winter et al.).

Cynthia Perry and colleagues used an ecological perspective to systematically review interventions targeting physical activity among adolescents. They found that most interventions were successful in promoting physical activity and that they targeted factors from multiple levels of the ecological domain, including individual (e.g., self-efficacy), social-network (e.g., peer support), and environmental (e.g., transportation) factors. The authors also emphasize the importance of grounding interventions in theory and conducting mediation analyses to specify components that contribute to a program's effectiveness.

Whether substance use (Palmer et al., 2009) or physical activity (Gordon-Larsen, Nelson, \& Popkin, 2004), behaviors and habits developed in the adolescent years have the potential to persist into adulthood. Therefore, we must continue to be diligent in researching adolescent development and applying what we learn to establish evidence-based prevention programs. As demonstrated by the articles herein, prevention programs aimed at changing adolescent behavior should be comprehensive in nature and target behaviors beyond the level of the individual.

\section{References}

Dent, C. W., Grube, J. W., \& Biglan, A. (2005). Community level alcohol availability and enforcement of possession laws as predictors of youth drinking. Preventive Medicine, 40, 355-362.

Gordon-Larsen, P., Nelson, M., \& Popkin, B. (2004). Longitudinal physical activity and sedentary behavior trends: Adolescence to adulthood. American Journal of Preventive Medicine, 27, 277-282.

Lac, A., \& Crano, W. D. (2009). Monitoring matters: Metaanalytic review reveals the reliable linkage of parental monitoring with adolescent marijuana use. Perspectives on Psychological Science, 4, 578-586.

Palmer, R. H. C., Young, S. E., Hopfer, C. J., Corley, R. P., Stallings, M. C., Crowley, T. J., et al. (2009). Developmental epidemiology of drug use and abuse in adolescence and young adulthood: Evidence of generalized risk. Drug and Alcohol Dependence, 102, 78-87.

Yancey, A. K., Siegel, J. M., \& McDaniel, K. L. (2002). Role models, ethnic identity, and health-risk behaviors in urban adolescents. Archives of Pediatrics and Adolescent Medicine, 156, 55-61. 